\title{
Riche-Cannieu Anastomosis: A Case Report
}

\author{
Eren Gozke ${ }^{\mathrm{a}, \mathrm{c}}$, Reyhan Gurer ${ }^{\mathrm{b}}$, Nurbanu Gurbuzer ${ }^{\mathrm{a}}$
}

\begin{abstract}
Riche-Cannieu anastomosis is a rare type of anomalous innervation. A 44 years old woman who has numbness her hands evaluated by electromyography. Nerve conduction studies showed dual innervation (mainly ulnar nerve) of abductor pollicis brevis muscle bilaterally. Needle electromyography of bilateral abductor pollicis brevis and abductor digiti minimi muscles are found as normal. U1nar to median anastomosis in the palm should be kept in mind in patient with unexpected electrophysiological results.
\end{abstract}

Keywords: Riche-Cannieu anastomosis; Anomalous innervation; Nerve conduction studies

\section{Introduction}

Anomalous ulnar to median nerve communications in the palm are known as Riche-Cannieu anastomosis (RCA). It is characterized as innervation of medial and lateral part of palm involving the deep branch of the ulnar nerve crossover to the recurrent branch of the median nerve in the hand. Clinical presentation of RCA can be three forms. All hand muscles can be innervated by ulnar nerve (all ulnar hand) or motor innervation can be dominantly provided by ulnar nerve and lastly some of median innervated muscles can be innervated by ulnar nerve [1-3].

Manuscript accepted for publication February 14, 2012

\footnotetext{
${ }^{a}$ Department of Neurology, FSM Teaching and Research Hospital, Istanbul, Turkey

${ }^{\mathrm{b}}$ Department of Neurology, Tuzla State Hospital, Istanbul, Turkey

${ }^{\mathrm{c}}$ Corresponding author: Eren Gozke. Email: egozke@hotmail.com
}

doi:10.4021/jmc576w

\section{Case Report}

A 44 years old right handed woman had been suffered from pain and numbness in the hands was examined. Her neurological examination was normal. There was no wasting or weakness of the hand muscles, nor sensory impairment. The pain was dull in character, continuing all day long. Phallen and Tinel signs were negative and peripheral nerves were normal by palpation.

Nerve conduction studies (NCS) and needle electromyography was performed using standard techniques. Skin temperature was kept above $32{ }^{\circ} \mathrm{C}$. Motor nerve conduction studies were obtained by recording compound muscle action potential (CMAP) responses from abductor pollicis brevis (APB) and abductor digit minimi (ADM) muscles following stimulation of median and ulnar nerves. Orthodromic sensory responses from the median and ulnar nerves were obtained from 2 nd and 5 th digits by using ring electrodes. Left median nerve motor conduction studies recorded from APB muscles obtained by stimulating the nerve at the wrist showed unusual bifid appearance with a small amplitude. Also stimulating the nerve at the elbow showed a similar appearance and amplitude so this also excludes Martin Gruber anastomosis. Recording from APB, this time ulnar nerve was stimulated at the wrist and cubital tunnel surprisingly responses with normal CMAP amplitude and latencies obtained (Fig. 1). Recording from ADM ulnar motor nerve conduction velocities were normal. After median nerve stimulation no response was recorded from ADM muscle. Similar findings were obtained on the right hand, though mildly higher CMAP amplitudes in right median nerve in comparison to left median nerve. Normal sensory nerve action potentials (SNAPs) were recorded from median and ulnar nerves bilaterally. Needle electromyography of APB, ADM, biceps, triceps and deltoid muscles were normal. All these findings pointed out dual innervation of APB (ulnar dominance), and RCA with pure motor innervation was diagnosed.

\section{Discussion}

Riche-Cannieu anastomosis is seen rarely than Martin-Gr- 


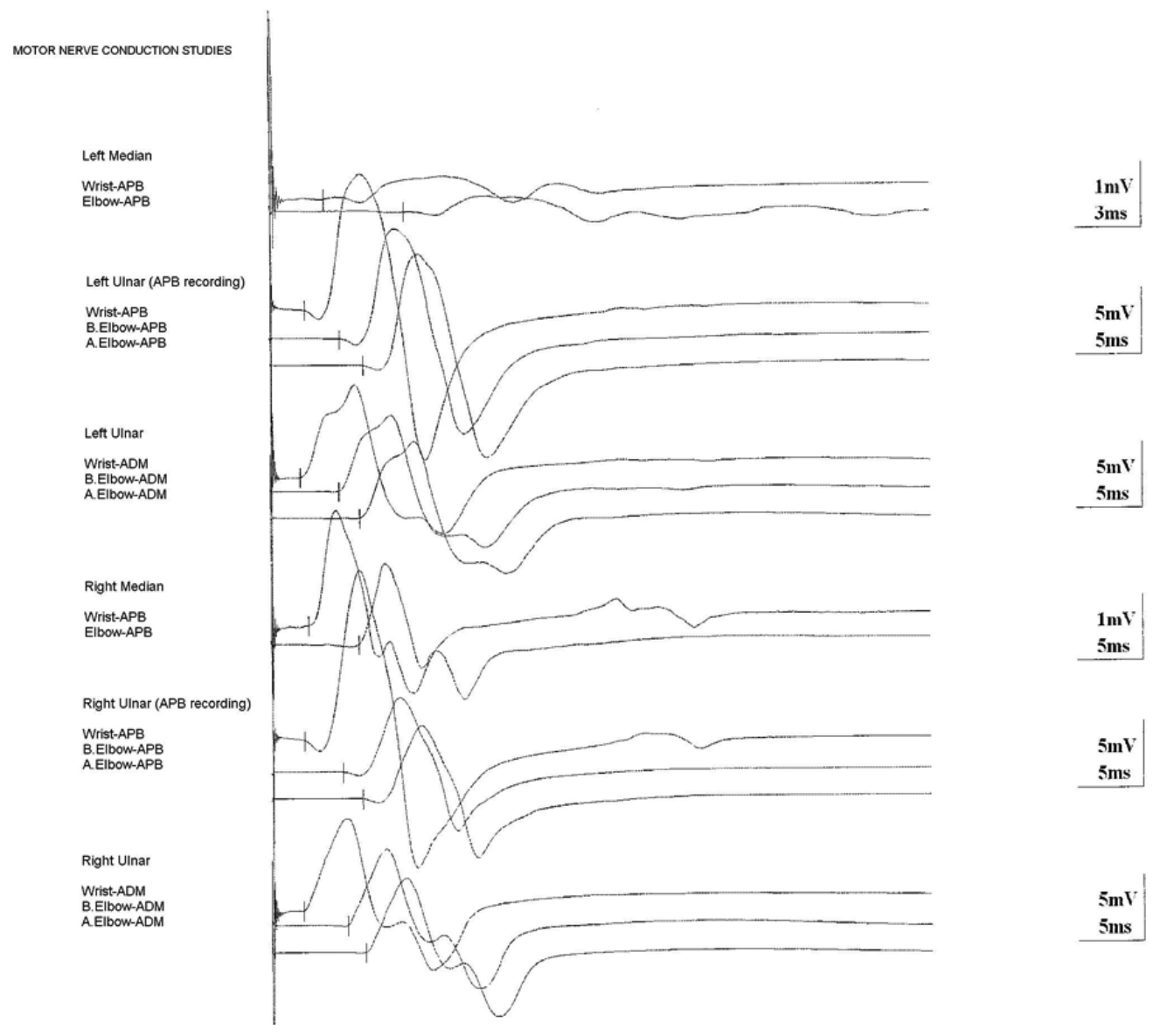

Figure 1. The findings of motor nerve conduction studies of the patient.

uber anastomosis (MGA) and its neurophysiologic features are known less than other anastomosis. So electromyographer must be very careful when interpretating these anomalous communications. Otherwise there may be misdiagnosis, as a case Saperstein and King reported ulnar neuropathy and RCA co-existence incorrectly diagnosed as motor neuron disease [4]. If the hand muscles are clinically intact but electrophysiological examination shows a severe median nerve lesion in the forearm, RCA should be suspected. Especially in surgical intervention, correct diagnose is very important. The definite reason of anastomosis is not known, but considered as developmental origin, also there are familial cases presenting RCA, these indicate autosomal dominant inheritance [5].

In conclusion, RCA should be excluded in patients who have numbness in hands, especially when extraordinary nerve conduction studies findings present.

\section{Conflict of Interest}

No benefits in any form have been received or will be received related directly or indirectly to the subject of this article.

\section{References}

1. Ganes T. Complete ulnar innervation of the thenar muscles combined with normal sensory fibres in a subject with no peripheral nerve lesion. Electromyogr Clin Neurophysiol. 1992;32(10-11):559-563.

2. Bolukbasi O, Turgut M, Akyol A. Ulnar to median nerve anastomosis in the palm (Riches-Cannieu anastomosis). Neurosurg Rev. 1999;22(2-3):138-139.

3. Kim BJ, Date ES, Lee SH, Lau EW, Park MK. Unilateral all ulnar hand including sensory without forearm communication. Am J Phys Med Rehabil. 2004;83(7):569573.

4. Saperstein DS, King RB. Motor neuron presentation of an ulnar neuropathy and Riche-Cannieu anastomosis. Electromyogr Clin Neurophysiol. 2000;40(2):119-122.

5. Boland RA, Krishnan AV, Kiernan MC. Riche-Cannieu anastomosis as an inherited trait. Clin Neurophysiol. 2007;118(4):770-775. 